\title{
Integrated guidance and control design method based on finite-time state observer
}

\author{
MA Ping, ZHANG Denghui, WANG Songyan, and CHAO Tao*
}

Control and Simulation Center, Harbin Institute of Technology, Harbin 150080, China

\begin{abstract}
A composited integrated guidance and control (IGC) algorithm is presented to tackle the problem of the IGC design in the dive phase for the bank-to-turn (BTT) vehicle with the inaccuracy information of the line-of-sight (LOS) rate. For the sake of theoretical derivation, an IGC model in the pitch plane is established. The high-order finite-time state observer (FTSO), with the LOS angle as the single input, is employed to reconstruct the states of the system online. Besides, a composited IGC algorithm is presented via the fusion of back-stepping and dynamic inverse. Compared with the traditional IGC algorithm, the proposed composited IGC method can attenuate effectively the design conservation of the flight control system, while the LOS rate is mixed with noise. Extensive experiments have been performed to demonstrate that the proposed approach is globally finite-time stable and strongly robust against parameter uncertainty.
\end{abstract}

Keywords: integrated guidance and control (IGC), finite-time state observer (FTSO), back-to-turn (BTT) vehicle, composited control.

DOI: $10.21629 / J S E E .2018 .06 .12$

\section{Introduction}

The traditional double-loops method is to design the guidance subsystem and control subsystem separately and then integrate them. In the terminal attack stage of vehicle against ground targets, the time constant of the guidance subsystem will be decreasing with the rapid decrease of relative vehicle-target distance. Consequently, the time constants of guidance and control system may not satisfy the original hypothesis [1]. Hence, the system performance degrades seriously.

Integrated guidance and control (IGC) is a new, feasible solution to deal with this problem. In IGC, the dynamic of angle of attack serves as a bridge to integrate the guidance and control subsystems. Therefore, IGC takes adequately advantage of the inherent system performance to guarantee a more optimal flight trajectory. In addition,

Manuscript received September 26, 2017.

*Corresponding author.

This work was supported by the National Natural Science Foundation of China (61627810; 61790562; 61403096).
IGC can assign reasonably the control ability of the vehicle and reduce effectively excessive control. Accordingly, the integrated design has attracted significant attention in recent decades [2-8]. Several advanced control methods have been investigated for the IGC system, including $\theta-D$ method [9], feedback linearization [10], predictive control [11], small gain [12], the state-dependent Riccatti equation [13], sliding mode control [13-15], dynamic surface control [16-18] and finite-time control [19-21]. To be more specific, Shima [22] chose the differential gamebased zero-effort miss (ZEM) distance as the sliding surface and designed a novel sliding-mode-based integrated algorithm. Two typical traditional double-loop methods are employed to validate the merit of the integrated algorithm in several endgame interception engagements. Yan [23] developed a small-gain theorem-based IGC algorithm for vehicle manipulated by both canard and tail controls. The input-to-state stability (ISS) theory is used to calculate the angle of attack and pitch rate commands, which generates the desired aerodynamic lift to realize accurate interception to a maneuvering target. Hou [24] aimed at the three channels independence IGC model to put forward a robust multi-channels IGC design approach and the adaptive dynamic surface technique was addressed to cope with these unmatched uncertainties. Extensive experiments show that the proposed design method can guarantee the accuracy strike to a ground fixed target. Nevertheless, it chose the side-to-turn (STT) vehicle as the subject for study.

The design of guidance law for hypersonic vehicles in the dive phase requires accurate measurement of the lineof-sight (LOS) rate. Unfortunately, it is usually impossible to detect via the radar seeker. Meanwhile, it is inevitable to introduce error, caused by computational methods or attitude rate in the process of extracting the LOS rate via the infrared seeker. To improve attack accuracy, stateestimation acts as an available solution to refactor the LOS rate. Guvfil [25] and Chwa et al. [26] respectively adopted the Kalman filter and the nonlinear observer to obtain the 
high-order derivatives of the LOS rate. As a result, the design of the controller does not have to depend on the LOS rate and its high-order derivatives. However, this solution has not taken full advantage of the system information. Besides, another scheme is to employ the filter, combined with the extended state observer to obtain the noise-free LOS rate. In this way, the noise perturbation can only be reduced but not eliminated, and the time constant of the filter has the tremendous impact on the precision of the filter. On account of the better convergence performance and anti-disturbance attribution [27], the non-smooth control theory has been used to design the robust controller in various nonlinear systems, e.g., nonlinear cascaded system [28, 29], switched stochastic nonlinear system [30].

The contribution of this paper is developing a composited finite-time state observer-based (FTSO) IGC design method for the bank-to-turn (BTT) vehicle against fixed ground targets. The high-order FTSO, with LOS as the single input, can make full use of the system information to estimate the LOS rate in real time. The composited IGC algorithm can attenuate effectively the design conservation and reduce excessive control. Simulation results verify the high precision endgame performance of the proposed IGC design method and strong robustness in spite of aerodynamic coefficient uncertainty and small-range target maneuver.

The remainder of this paper is organized as follows: In the next section, an IGC model in the pitch plane is formulated. Then a composited FTSO-based IGC algorithm is designed in detail. Extensive experiments have been performed in Section 4. Finally the conclusion is arrived at.

\section{Problem formulation}

In this section, a longitudinal strict-feedback IGC design model is formulated and some essential fundamental preliminaries are provided.

\subsection{IGC design model}

The longitudinal nonlinear dynamic [31] is described as follows:

$$
\begin{gathered}
\dot{\alpha}=\omega_{z}-\frac{57.3 q S_{r e f} c_{y}^{\alpha}}{m V} \alpha+\frac{g \cos \theta}{V} \\
\dot{\omega}_{z}=\frac{57.3 q L S_{r e f} m_{z}^{\alpha}}{J_{z}} \alpha+\frac{q S_{r e f} L^{2} m_{z}^{\omega_{z}}}{J_{z} V} \omega_{z}+ \\
\frac{57.3 q L S_{r e f} m_{z}^{\delta_{z}}}{J_{z}} \delta_{z}
\end{gathered}
$$

where $V$ is the velocity of vehicle, $\theta$ is the flight path angle, $\alpha$ is the angle of attack, $\omega_{z}$ is the rotating rate along the pitch axis, $L$ is the reference length, $S_{\text {ref }}$ is the reference area, $J_{z}$ is the moment of inertia about the pitch axis, $\delta_{z}$ is the elevator deflection angle, $q$ denotes the dynamic pressure, $c_{y}^{\alpha}$ represents the nominal values of the lift force coefficient derivative with respect to the angle of attack, $m_{z}^{\alpha}, m_{z}^{\omega_{z}}$ and $m_{z}^{\delta_{z}}$ are respectively the nominal values of the pitch moment coefficient derivatives with respect to the angle of attack, the rotating rate along the pitch axis and the elevator deflection angle.

The nonlinear engagement kinematics, regardless of the lateral plane, is given by

$$
\begin{gathered}
\ddot{\lambda}_{D}=-\frac{2 \dot{r}}{r} \dot{\lambda}_{D}-\frac{57.3 q S_{r e f} c_{y}^{\alpha}}{m r} \alpha+ \\
\frac{g \cos \theta \cos \left(\lambda_{D}-\theta\right)}{r}
\end{gathered}
$$

where $r$ and $\lambda_{D}$ are the relative distance and the LOS angle, respectively.

The longitudinal IGC design model can be got by means of merging sub-dynamic (1)-(3).

$$
\left\{\begin{array}{l}
\dot{x}_{1}=x_{2} \\
\dot{x}_{2}=a_{21} x_{2}+a_{22} x_{3}+G_{1} \\
\dot{x}_{3}=a_{31} x_{3}+x_{4}+G_{2} \\
\dot{x}_{4}=a_{41} x_{3}+a_{42} x_{4}+b u \\
y=x_{1}
\end{array}\right.
$$

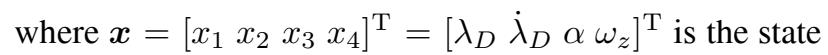
vector, $u=\delta_{z}$ denotes the input of system, $y=\lambda_{D}$ represents the output of system. The other nonlinear terms and gain coefficients of state variables are shown as follows:

$$
\begin{gathered}
G_{1}=\frac{g \cos \theta \cos \left(\lambda_{D}-\theta\right)}{r}, \quad G_{2}=\frac{g \cos \theta}{V} \\
a_{21}=-\frac{2 \dot{r}}{r}, \quad a_{22}=-\frac{57.3 q S_{r e f} c_{y}^{\alpha}}{m r} \\
a_{31}=-\frac{57.3 q S_{r e f} c_{y}^{\alpha}}{m V}, \quad a_{41}=\frac{57.3 q L S_{r e f} m_{z}^{\alpha}}{J_{z}} \\
b=\frac{57.3 q L S_{r e f} m_{z}^{\delta_{z}}}{J_{z}} \quad a_{42}=\frac{q L^{2} S_{r e f} m_{z}^{\omega}}{J_{z} V} .
\end{gathered}
$$

Furthermore, the dynamic model (4) can be rewritten as the strict-feedback nonlinear system represented by

$$
\left\{\begin{array}{l}
\dot{x}_{1}=f_{1}\left(t, x_{1}\right)+x_{2} \\
\dot{x}_{2}=f_{2}\left(t, x_{1}, x_{2}\right)+a_{22} x_{3} \\
\dot{x}_{3}=f_{3}\left(t, x_{1}, x_{2}, x_{3}\right)+x_{4} \\
\dot{x}_{4}=f_{4}\left(t, x_{1}, x_{2}, x_{3}, x_{4}\right)+b u \\
y=x_{1}
\end{array}\right.
$$

where $f_{1}\left(t, x_{1}\right)=0, f_{2}\left(t, x_{1}, x_{2}\right)=a_{21} x_{2}+G_{1}$, $f_{3}\left(t, x_{1}, x_{2}, x_{3}\right)=a_{31} x_{3}+G_{2}, f_{4}\left(t, x_{1}, x_{2}, x_{3}, x_{4}\right)=$ $a_{41} x_{3}+a_{42} x_{4}$.

The design objective is to propose a composited robust IGC algorithm to ensure the accurate strike against the ground fixed target, while the LOS rate is mixed with uncertainty. 


\subsection{Fundamental preliminaries}

For the ease of the design of high-order FTSO, several necessary assumptions and lemmas are presented.

Assumption 1 There are a smooth function $\eta(z)>0$ and constants $\underline{\sigma}>0, \bar{\sigma}>0$, which guarantee $\phi(t, z)$ bounded

$$
\begin{gathered}
0<\underline{\sigma} \eta(z) \leqslant \phi_{j}(t, z) \leqslant \bar{\sigma} \eta(z) \\
\forall t \geqslant 0, z \in \mathbf{R} .
\end{gathered}
$$

where $j=1,2, \ldots, n$.

Assumption 2 There exist positive constants $\beta_{j}$ and $\omega \in(-1 / n, 0)$ to guarantee the following inequality valid for $\forall t, z_{j}, \tilde{z}_{j}, u \in \mathbf{R}$ :

$$
\begin{gathered}
\left|f_{j}\left(t, z_{1}, z_{2}, \ldots, z_{j}, u\right)-f_{j}\left(t, z_{1}, \tilde{z}_{2}, \ldots, \tilde{z}_{j}, u\right)\right| \leqslant \\
\beta_{j} \phi_{j}(t, z) \sum_{k=2}^{j}\left|z_{k}-\tilde{z}_{k}\right|{ }^{\left(m_{j}+\omega\right) / m_{k}}
\end{gathered}
$$

where $m_{j}=1+(j-1) \omega(j=2,3, \ldots, n)$.

Assumption 3 The LOS angle could be obtained fairly accurately via the seeker in the dive phase.

Lemma 1 [32] Suppose that there exist a positive definite and continuously differentiable function $V(x)$ on the region $U \subset \mathbf{R}^{n}$ around the origin, real numbers $c>0$ and $\gamma \in(0,1)$. When the term $\dot{V}(x)+c V^{\gamma}(x)$ is negative semi-definite, the origin is a finite-time-stable equilibrium.

Lemma 2 [33] For $z \in \mathbf{R}, w \in \mathbf{R}$, there exist real numbers $s>0, t>0$ and real-valued function $\vartheta(z, w)>0$. Then

$$
|z|^{s}|w|^{t} \leqslant \frac{s}{s+t} \vartheta|z|^{s+t}+\frac{s}{s+t} \vartheta^{-s / t}|w|^{s+t} .
$$

Lemma 3 [30] For $z \in \mathbf{R}, w \in \mathbf{R}$ and a ratio of positive odd integers $k \geqslant 1$, the following inequalities hold:

(i) $\left|z^{k}-w^{k}\right| \leqslant k|z-w|\left(|z|^{k-1}+|w|^{k-1}\right) \leqslant c \mid z-$ $\left.w\right|^{k}+c|z-w||w|^{k-1}, \quad c=k\left(2+2^{k-2}\right)$;

(ii) $\left|z^{k}-w^{k}\right| \geqslant 2^{1-k}|z-w|^{k}$;

(iii) $|z+w|^{k} \leqslant 2^{k-1}\left|z^{k}+w^{k}\right|$.

Lemma 4 The following inequality is valid:

$$
\left(\sum_{i=1}^{n}\left|\beta_{i}\right|^{2}\right)^{\eta / 2} \leqslant \sum_{i=1}^{n}\left|\beta_{i}\right|^{\eta}
$$

when $\eta \in(0,2)$.

Lemma 5 [34] Assume $Q: \mathbf{R}^{n} \rightarrow \mathbf{R}$ is a homogeneous function of degree $\omega$ as for dilation weight $\Psi$. Hence, the following facts meet:

(i) The homogeneous of degree about the partial differential term $\frac{\partial Q}{\partial z_{i}}$ is $\omega-\rho_{i}$ and $\rho_{i}$ acts as the homogeneous weight of $z_{i}$. (ii) The condition $Q(x) \leqslant \varpi W(x)$ would be satisfied, when the positive definite function $W$ (defined on $\left.U \subset \mathbf{R}^{n}\right)$ is homogeneous of degree $\omega$ as for dilation $\Psi$, where $\varpi>0$.

\section{IGC design}

The high-order FTSO, with LOS angle as the single input, is presented to make full use of the system information to reconstruct the LOS rate. The composited IGC method is developed to tackle the challenge caused by the inaccuracy measurement of the LOS rate. Besides, the corresponding strict proof process of stability is provided.

\subsection{FTSO}

Theorem 1 When Assumptions 1-3 are satisfied and the proper constants $k_{i}$ are selected, the proposed highorder FTSO (6) for nonlinear system (5) can ensure the estimations $\left(\hat{x}_{1}, \ldots, \hat{x}_{4}\right)$ to track well the actual state variables $\left(x_{1}, \ldots, x_{4}\right)$ in a finite time.

$$
\left\{\begin{aligned}
\dot{\hat{x}}_{1}= & \hat{x}_{2}+f_{1}\left(t, x_{1}, u\right)+ \\
& L k_{1} \operatorname{sig}^{m_{2}}\left(x_{1}-\hat{x}_{1}\right) \\
\dot{\hat{x}}_{2}= & a_{22} \hat{x}_{3}+f_{2}\left(t, x_{1}, \hat{x}_{2}, u\right)+ \\
& a_{22} L^{2} k_{2} \operatorname{sig}^{m_{3}}\left(x_{1}-\hat{x}_{1}\right) \\
\dot{\hat{x}}_{3}= & \hat{x}_{4}+f_{3}\left(t, x_{1}, \hat{x}_{2}, \hat{x}_{3}, u\right)+ \\
& L^{3} k_{3} \operatorname{sig}^{m_{4}}\left(x_{1}-\hat{x}_{1}\right) \\
\dot{\hat{x}}_{4}= & b u+f_{4}\left(t, x_{1}, \hat{x}_{2}, \hat{x}_{3}, \hat{x}_{4}, u\right)+ \\
& b L^{4} k_{4} \operatorname{sig}^{m_{5}}\left(x_{1}-\hat{x}_{1}\right)
\end{aligned}\right.
$$

where $\omega \in(1 / n, 0)$ and $m_{i}=(i-1) \omega+1, i=$ $1,2, \ldots, n+1$.

Proof

(i) To begin with, the high-order FTSO (7) is designed for the following fourth-order integral system:

$$
\left\{\begin{array}{l}
\dot{x}_{1}=x_{2} \\
\dot{x}_{2}=a_{22} x_{3} \\
\dot{x}_{3}=x_{4} \\
\dot{x}_{4}=b u \\
y=x_{1}
\end{array}\right.
$$

which can guarantee availably the estimations $\left(\hat{x}_{2}, \hat{x}_{3}, \hat{x}_{4}\right)$ to track well the actual state variables $\left(x_{2}, x_{3}, x_{4}\right)$ of the integral system in a finite time, when Assumption 1 is met and appropriate parameters $k_{i}$ are chosen.

$$
\left\{\begin{array}{l}
\dot{\hat{x}}_{1}=\hat{x}_{2}+k_{1} \operatorname{sig}^{m_{2}}\left(x_{1}-\hat{x}_{1}\right) \\
\dot{\hat{x}}_{2}=a_{22} \hat{x}_{3}+a_{22} k_{2} \operatorname{sig}^{m_{3}}\left(x_{1}-\hat{x}_{1}\right) \\
\dot{\hat{x}}_{3}=\hat{x}_{4}+k_{3} \operatorname{sig}^{m_{4}}\left(x_{1}-\hat{x}_{1}\right) \\
\dot{\hat{x}}_{4}=b u+b k_{4} \operatorname{sig}^{m_{5}}\left(x_{1}-\hat{x}_{1}\right)
\end{array}\right.
$$

where $\omega \in(1 / n, 0)$ and $m_{i}=(i-1) \omega+1, i=$ $1,2, \ldots, n+1$. 
The errors of the FTSO are defined as $\bar{e}_{i}=x_{i}-\hat{x}_{i}(i=$ $1, \ldots, 4)$. Then, one can obtain that

$$
\left\{\begin{array}{l}
\dot{\bar{e}}_{1}=\bar{e}_{2}-k_{1} \operatorname{sig}^{m_{2}}\left(\bar{e}_{1}\right) \\
\dot{\bar{e}}_{2}=a_{22} \bar{e}_{3}-a_{22} k_{2} \operatorname{sig}^{m_{3}}\left(\bar{e}_{1}\right) \\
\dot{\bar{e}}_{3}=\bar{e}_{4}-k_{3} \operatorname{sig}^{m_{4}}\left(\bar{e}_{1}\right) \\
\dot{\bar{e}}_{4}=-b k_{4} \operatorname{sig}^{m_{5}}\left(\bar{e}_{1}\right)
\end{array} .\right.
$$

Then, some efforts are made to validate the errors dynamic (8) of FTSO globally finite-time stable, when the proper parameters $k_{i}$ are chosen.

Applying the nonlinear mapping,

$$
\left\{\begin{array}{l}
e_{1}=\operatorname{sig}\left(\bar{e}_{1}\right) \\
e_{2}=\operatorname{sig}\left(\bar{e}_{2}\right) / k_{1} \\
e_{3}=\operatorname{sig}\left(\bar{e}_{3}\right) / k_{2} \\
e_{4}=\operatorname{sig}\left(\bar{e}_{4}\right) / k_{3}
\end{array}\right.
$$

the dynamic of the errors system (8) could be translated into

$$
\left\{\begin{array}{l}
\dot{e}_{1}=l_{1}\left(e_{2}-e_{1}^{m_{2}}\right) \\
\dot{e}_{2}=a_{22} l_{2}\left(e_{3}-e_{1}^{m_{3}}\right) \\
\dot{e}_{3}=l_{3}\left(e_{4}-e_{1}^{m_{4}}\right) \\
\dot{e}_{4}=-b l_{4} e_{1}^{m_{5}}
\end{array}\right.
$$

where $l_{1}=k_{1}, l_{2}=k_{2} / k_{1}, l_{3}=k_{3} / k_{2}, l_{4}=k_{4} / k_{3}$.

Next, it is necessary to indicate that the states of the translated system (10) are globally finite-time stable, while the proper parameters $l_{i}$ are chosen.

The nonlinear mapping is defined as

$$
G_{i, j}\left(e_{j+1}, e_{j}\right)=e_{j+1}^{\frac{m_{i+1}}{m_{j+1}}}-e_{j}^{\frac{m_{i+1}}{m_{j}}}
$$

where $i=1,2, \ldots, n, j=1, \ldots, i$ and $e_{5}=0$. On account of the above mapping and the attribute of $m_{i}$, the following propositions hold.

Proposition 1 The following facts hold:

(i) $\sum_{j=1}^{i} G_{i, j}=e_{i+1}-e_{1}^{m_{i+1}}, i=1,2, \ldots, n$;

(ii) $\left|G_{i, j}\right| \leqslant 2^{1-\frac{m_{i+1}}{m_{j+1}}}\left|G_{j, j}\right|^{\frac{m_{i+1}}{m_{j+1}}}, j=1,2, \ldots, n-$ $1, i>j$;

(iii) $\left|G_{i, j}\right| \leqslant \lambda\left|G_{j, j}\right|\left(\left|G_{j, j}\right|^{\frac{m_{i+1}}{m_{j+1}}-1}+\left|e_{j+1}\right|^{\frac{m_{i+1}}{m_{j+1}}-1}\right)$, $j=2,3, \ldots, n, i<j$, for constant $\lambda>0$.

Proposition 2 There is a positive constant $\xi$ to guarantee the inequality $\left|e_{k}\right|^{\frac{2+\omega}{m_{k}}} \leqslant \xi \sum_{j=k}^{n}\left|G_{j, j}\right|^{\frac{2+\omega}{m_{j+1}}}$ valid, where $k=1,2, \ldots, n$.

Proposition 3 There exists a constant $\varsigma$ to guarantee the following inequality valid:

$$
\sum_{k=n-i+1}^{n} \frac{\partial Q_{n-i+1}}{\partial e_{k}} \varphi_{k} l_{k} G_{k, n-i} \leqslant
$$

$$
\frac{1}{2} \sum_{j=n-i+1}^{n} \phi_{j} G_{j, j}^{\frac{2+\tau}{m_{j+1}}}+\varsigma \phi_{n-i} G_{n-i, n-i}^{\frac{2+\tau}{n-i+1}} .
$$

Proposition 4 The following inequality

$$
\begin{gathered}
\dot{V}_{n-i} \leqslant \frac{1}{2} \sum_{j=n-i+1}^{n} \phi_{j} G_{j, j}^{\frac{2+\tau}{m_{j+1}}}-\left[2^{1-\frac{2-m_{n-i}}{m_{n-i+1}}} l_{n-i}-\xi\right] . \\
\phi_{n-i} G_{n-i, n-i}^{\frac{2+\tau}{m_{n-i+1}}}
\end{gathered}
$$

is valid, when the proper parameter $\xi$ is selected.

Based on Proposition 1, the system (10) can be translated into

$$
\left\{\begin{array}{l}
\dot{e}_{1}=l_{1} G_{1,1} \\
\dot{e}_{2}=a_{22} l_{2} \sum_{i=1}^{2} G_{2, i} \\
\dot{e}_{3}=l_{3} \sum_{i=1}^{3} G_{3, i} \\
\dot{e}_{4}=b l_{4} \sum_{i=1}^{4} G_{4, i}
\end{array} .\right.
$$

The bottom-up inductive skill is adopted to validate that the error system (12) is globally finite-time stable, when the proper parameters $l_{i}$ are chosen.

Step 1 The following partial system is investigated firstly:

$$
\dot{e}_{4}=b l_{4} G_{4,4}
$$

which describes the error dynamic of $\omega_{z}$.

The appropriate Lyapunov candidate function, which is continuously differentiable and positive definite, could be adopted.

$$
Q_{4}\left(e_{4}\right)=V_{4}=\int_{0}^{e_{4}}\left(s^{\frac{2-m_{4}}{m_{4}}}\right) \mathrm{d} s=\frac{m_{4}}{2} e_{4}^{\frac{2}{m_{4}}}
$$

The following inequality scaling is effective:

$$
\begin{gathered}
\left.\dot{Q}_{4}\left(e_{4}\right)\right|_{(16)}=e_{4}^{\frac{2-m_{4}}{m_{4}}} \dot{e}_{4}= \\
-b l_{4} G_{4,4}^{\frac{2-m_{4}}{m_{5}}} G_{4,4} \leqslant-4 b G_{4,4}^{\frac{2+\tau}{m_{5}}}
\end{gathered}
$$

when the appropriate parameter $l_{4} \geqslant 4$ is selected.

Step 2 The following partial system is researched:

$$
\left\{\begin{array}{l}
\dot{e}_{3}=l_{3} G_{3,3} \\
\dot{e}_{4}=b l_{4} \sum_{i=3}^{4} G_{4, i}
\end{array}\right.
$$

The following Lyapunov candidate function is employed:

$$
Q_{3}\left(e_{3}, e_{4}\right)=Q_{4}+V_{3}
$$

where $V_{3}=\int_{e_{4}^{m_{3} / m_{4}}}^{e_{3}}\left(s^{\frac{2-m_{3}}{m_{3}}}-e_{4}^{\frac{2-m_{3}}{m_{4}}}\right) \mathrm{d} s$. Taking the 
derivative of $Q_{3}\left(e_{3}, e_{4}\right)$ and integrating with the error dynamic (16), one can address that

$$
\left.\dot{Q}_{3}\right|_{(16)}=\left.\dot{Q}_{4}\right|_{(16)}+\left.\dot{V}_{3}\right|_{(16)} .
$$

The inequality of the scaling transformation is applied into (18). Based on the conclusion (15) in the first step and the property $\frac{\partial Q_{4}}{\partial e_{4}}=-G_{4,4}^{\frac{2-m_{4}}{m_{5}}}$, one can conclude that

$$
\begin{gathered}
\left.\dot{Q}_{4}\right|_{(16)}=-b l_{4} G_{4,4}^{\frac{2+\omega}{m_{5}}}-G_{4,4}^{\frac{2+\omega}{m_{5}}} b l_{4} G_{4,3} \leqslant \\
-4 b G_{4,4}^{\frac{2+\omega}{m_{5}}}-b l_{4} G_{4,4}^{\frac{2+\omega}{m_{5}}} G_{4,3} .
\end{gathered}
$$

Based on Proposition 1 and Lemma 2, it is obvious that

$$
\begin{gathered}
l_{4}\left|G_{4,4}^{\frac{2-m_{4}}{m_{5}}} G_{4,3}\right| \leqslant l_{4}\left|G_{4,4}\right|^{\frac{2-m_{4}}{m_{5}}} 2^{\frac{-\omega}{m_{4}}}\left|G_{3,3}\right|^{\frac{m_{5}}{m_{4}}} \leqslant \\
\frac{1}{2} G_{4,4}^{\frac{2+\omega}{m_{5}}}+\kappa_{1} G_{3,3}^{\frac{2+\omega}{m_{4}}}
\end{gathered}
$$

where $\kappa_{1}>0$. Integrating (20) and (19), the following inequality can be got

$$
\left.\dot{Q}_{4}\right|_{(15)} \leqslant-b\left(4-\frac{1}{2}\right) G_{4,4}^{\frac{2+\omega}{m_{5}}}+b \kappa_{1} G_{3,3}^{\frac{2+\omega}{m_{4}}} .
$$

In addition, taking the derivative of $V_{3}$ and integrating with the error dynamic (16), it is clear that

$$
\begin{gathered}
\left.\dot{V}_{3}\right|_{(16)}=-\frac{2-m_{3}}{m_{4}} e_{4}^{\frac{2-m_{3}}{m_{4}}-1} \dot{e}_{4}\left(e_{3}-e_{4}^{\frac{m_{3}}{m_{4}}}\right)+ \\
\left.\frac{2-m_{3}}{m_{4}} G_{4,4}^{\frac{2-m_{3}-m_{4}}{m_{3}}}-e_{4}^{\frac{2-m_{3}}{m_{4}}}\right) \dot{e}_{3}= \\
\left(l_{4}\left(G_{4,3}+G_{4,4}\right) G_{2,3}+\right. \\
\left.\frac{2-m_{3}}{m_{3}}-e_{4}^{\frac{2-m_{3}}{m_{4}}}\right) l_{3} G_{3,3} .
\end{gathered}
$$

Then, the inequality scaling technique is also used to transform the every member in (22). Above all, it can be obtained based on Proposition 1 (iii) that

$$
\begin{gathered}
\left|G_{2,3}\right| \leqslant \lambda\left|G_{3,3}\right|^{\frac{m_{3}}{m_{4}}}+\lambda\left|G_{3,3}\right|\left|e_{4}\right|^{\frac{m_{3}-m_{4}}{m_{4}}} \leqslant \\
\lambda\left|G_{3,3}\right|^{\frac{m_{3}}{m_{4}}}+\lambda\left|G_{3,3}\right|\left|G_{4,4}\right|^{\frac{m_{3}-m_{4}}{m_{5}}} .
\end{gathered}
$$

According to (23), one can acquire that

$$
\begin{gathered}
\frac{2-m_{3}}{m_{4}} G_{4,4}^{\frac{2-m_{3}-m_{4}}{m_{5}}} l_{4} G_{4,4} G_{2,3} \leqslant \\
\frac{2-m_{3}}{m_{4}} l_{4} \lambda\left|G_{4,4}^{\frac{1}{m_{5}}}\right|^{2-m_{3}+\omega}\left|G_{3,3}^{\frac{1}{m_{4}}}\right|^{m_{3}}+ \\
\frac{2-m_{3}}{m_{4}} l_{4} \lambda\left|G_{4,4}^{\frac{1}{m_{5}}}\right|^{2-m_{4}+\omega}\left|G_{3,3}^{\frac{1}{m_{4}}}\right|^{m_{4}} .
\end{gathered}
$$

Based on Lemma 2, the inequality (24) can be translated into

$$
\frac{-m_{3}+2}{m_{4}} G_{4,4}^{\frac{-m_{4}-m_{3}+2}{m_{5}}} l_{4} G_{4,4} G_{2,3} \leqslant \frac{1}{4} G_{4,4}^{\frac{2+\omega}{m_{5}}}+\kappa_{2} G_{3,3}^{\frac{2+\omega}{m_{4}}}
$$

where $\kappa_{2}>0$. Analogously, integrating Proposition 1 with Lemma 2, the inequality (23) can be translated into

$$
\begin{gathered}
\frac{2-m_{3}}{m_{4}} G_{4,4}^{\frac{2-m_{3}-m_{4}}{m_{5}}} l_{4} G_{2,3} G_{4,3} \leqslant \\
\frac{2-m_{3}}{m_{4}} G_{4,4}^{\frac{2-m_{3}-m_{4}}{m_{5}}} 2^{1-\frac{m_{5}}{m_{4}}}\left|G_{3,3}\right|^{\frac{m_{5}}{m_{4}}} . \\
l_{4} \lambda\left(\left|G_{3,3}\right|^{\frac{m_{3}}{m_{4}}}+\left|G_{3,3}\right|\left|G_{4,4}\right|^{\frac{m_{3}-m_{4}}{m_{5}}}\right) \leqslant \\
\frac{1}{4} G_{4,4}^{\frac{2+\omega}{m_{5}}}+\kappa_{3} G_{3,3}^{\frac{2+\omega}{m_{4}}}
\end{gathered}
$$

where $\kappa_{3}>0$. Moreover, based on Lemma 3 and the property $\frac{2-m_{3}}{m_{4}}>1$, one can obtain that

$$
\begin{gathered}
\left(e_{3}^{\frac{2-m_{3}}{m_{3}}}-e_{4}^{\frac{2-m_{3}}{m_{4}}}\right) l_{3} G_{3,3}=\left(e_{3}^{\frac{2-m_{3}}{m_{3}}}-e_{4}^{\frac{2-m_{3}}{m_{4}}}\right) l_{3}\left(e_{4}-e_{3}^{\frac{m_{4}}{m_{3}}}\right)= \\
-l_{3}\left|e_{4}^{\frac{2-m_{3}}{m_{4}}}-e_{3}^{\frac{m_{4}}{m_{3}} \frac{2-m_{3}}{m_{4}}}\right|\left|e_{4}-e_{3}^{\frac{m_{4}}{m_{3}}}\right| \leqslant \\
-l_{3} 2^{\frac{m_{3}+m_{4}-2}{m_{4}}}\left|e_{4}-e_{3}^{\frac{m_{4}}{m_{3}}}\right|^{\frac{2+\omega}{m_{4}}}=-l_{3} 2^{\frac{m_{3}+m_{4}-2}{m_{4}}} G_{3,3}^{\frac{2+\omega}{m_{4}}} .
\end{gathered}
$$

Substituting (25)-(27) into (22) yields

$$
\left.\dot{V}_{3}\right|_{(16)} \leqslant \frac{1}{2} b G_{4,4}^{\frac{2+\omega}{m_{5}}}-\left(l_{3} 2^{\frac{m_{3}+m_{4}-2}{m_{4}}}-b \kappa_{2}-b \kappa_{3}\right) G_{3,3}^{\frac{2+\tau}{m_{4}}} \text {. }
$$

With the aid of (21) and (28), (18) turns into

$$
\begin{gathered}
\left.\dot{Q}_{3}\right|_{(16)} \leqslant-b(4-1) G_{4,4}^{\frac{2+\omega}{m_{5}}}-\left(l_{3} 2^{\frac{m_{3}+m_{4}-2}{m_{4}}}-\right. \\
\left.b \kappa_{1}-b \kappa_{2}-b \kappa_{3}\right) G_{3,3}^{\frac{2+\omega}{m_{4}}} .
\end{gathered}
$$

Based on Assumption $1\left(\phi_{4} / \phi_{3} \leqslant \bar{\sigma} / \underline{\sigma}\right)$, the following inequality is valid, when the proper parameter $\kappa_{3}$ is selected.

$$
l_{3} 2^{\frac{m_{3}+m_{4}-2}{m_{4}}}-\left(\kappa_{1}+\kappa_{2}+\kappa_{3}\right) \phi_{4} / \phi_{3} \geqslant 4-1
$$

Hence, it can be easily got that

$$
\dot{Q}_{3} \leqslant-(4-1)\left(b G_{4,4}^{\frac{2+\omega}{m_{5}}}+G_{3,3}^{\frac{2+\omega}{m_{4}}}\right) .
$$

Step 3 The following partial system is considered:

$$
\left\{\begin{array}{l}
\dot{e}_{2}=a_{22} l_{2} G_{2,2} \\
\dot{e}_{3}=l_{3} \sum_{i=2}^{3} G_{3, i} \\
\dot{e}_{4}=b l_{4} \sum_{i=2}^{4} G_{4, i}
\end{array}\right.
$$


The following Lyapunov candidate function is used:

$$
\left.Q_{2}\right|_{(32)}\left(e_{2}, e_{3}, e_{4}\right)=\left.Q_{3}\right|_{(32)}\left(e_{3}, e_{4}\right)+\left.V_{2}\right|_{(32)}
$$

where $V_{2}=\int_{e_{3}^{\frac{m_{2}}{m_{3}}}}^{e_{2}}\left(s^{\frac{2-m_{2}}{m_{2}}}-e_{3}^{\frac{2-m_{2}}{m_{3}}}\right) \mathrm{d} s$. Taking the derivative of $Q_{2}\left(e_{2}, e_{3}, e_{4}\right)$ and integrating with the error dynamic (32), one can obtain that

$$
\left.\dot{Q}_{2}\right|_{(32)}=\left.\dot{Q}_{3}\right|_{(32)}+\left.\dot{V}_{2}\right|_{(32)} .
$$

The inequality of the scaling transformation is applied into (34). Based on the conclusion (31), one can conclude that

$$
\begin{gathered}
\left.\dot{Q}_{3}\right|_{(32)}=\frac{\partial Q_{3}}{\partial e_{3}} \dot{e}_{3}+\frac{\partial Q_{3}}{\partial e_{4}} \dot{e}_{4} \leqslant \\
-3\left(b G_{4,4}^{\frac{2+\omega}{m_{5}}}+G_{3,3}^{\frac{2+\omega}{m_{4}}}\right)+ \\
\frac{\partial Q_{3}}{\partial e_{3}} l_{3} G_{3,2}+\frac{\partial Q_{3}}{\partial e_{4}} b l_{4} G_{4,2} .
\end{gathered}
$$

Based on Proposition 3, it is clear that

$$
\begin{gathered}
\frac{\partial Q_{3}}{\partial e_{4}} b l_{4} G_{4,2}+\frac{\partial Q_{3}}{\partial e_{3}} l_{3} G_{3,2} \leqslant \\
\frac{1}{2}\left(G_{3,3}^{\frac{2+\omega}{m_{4}}}+b G_{4,4}^{\frac{2+\omega}{m_{5}}}\right)+\rho_{1} a_{22} G_{2,2}^{\frac{2+\omega}{m_{3}}} .
\end{gathered}
$$

Substituting (36) into (35) yields

$$
\begin{gathered}
\left.\dot{Q}\left(e_{3}, e_{4}\right)\right|_{(32)} \leqslant-\left(3-\frac{1}{2}\right)\left(b G_{4,4}^{\frac{2+\omega}{m_{5}}}+\right. \\
\left.G_{3,3}^{\frac{2+\omega}{m_{4}}}\right)+\rho_{1} a_{22} G_{2,2}^{\frac{2+\omega}{m_{3}}} .
\end{gathered}
$$

Based on Proposition 4, it is got that

$$
\begin{gathered}
\left.\dot{V}_{2}\right|_{(32)} \leqslant \frac{1}{2}\left(b G_{4,4}^{\frac{2+\omega}{m_{5}}}+G_{3,3}^{\frac{2+\omega}{m_{4}}}\right)- \\
{\left[2^{1-\frac{2-m_{2}}{m_{3}}} l_{2}-\xi_{1}\right] a_{22} G_{2,2}^{\frac{2+\omega}{m_{3}}} .}
\end{gathered}
$$

Substituting (37) and (38) into (35) yields

$$
\begin{aligned}
\dot{Q}_{2} & \leqslant-\left(3-\frac{1}{2}-\frac{1}{2}\right)\left(b G_{4,4}^{\frac{2+\omega}{m_{5}}}+G_{3,3}^{\frac{2+\omega}{m_{4}}}\right)- \\
& -a_{22} G_{2,2}^{\frac{2+\omega}{m_{3}}}\left(2^{1-\frac{2-m_{2}}{m_{3}}} l_{2}-\rho_{1}-\xi_{1}\right) .
\end{aligned}
$$

The similar conclusion (40) can be gained when the proper parameter $l_{2}$ are chosen.

$$
\dot{W}_{2} \leqslant-2\left(b F_{4,4}^{\frac{2+\tau}{m_{5}}}+F_{3,3}^{\frac{2+\tau}{m_{4}}}+a_{22} F_{2,2}^{\frac{2+\tau}{m_{3}}}\right)
$$

Step 4 The whole system (12) is researched and the following Lyapunov candidate function is addressed

$$
\left.Q_{1}\right|_{(12)}=\left.Q_{2}\right|_{(12)}+V_{1}
$$

where $V_{1}=\int_{e_{2}^{\frac{m_{1}}{m_{2}}}}^{e_{1}}\left(s^{\frac{2-m_{1}}{m_{1}}}-e_{2}^{\frac{2-m_{1}}{m_{2}}}\right) \mathrm{d} s$. Taking the derivative of $Q_{1}$ and integrating with the error dynamic (12), one can get that

$$
\left.\dot{Q}_{1}\right|_{(12)}=\left.\dot{Q}_{2}\right|_{(12)}+\left.\dot{V}_{1}\right|_{(12)} .
$$

Similar to Step 3, it is obvious that

$$
\begin{gathered}
\left.\dot{Q}_{1}\right|_{(12)} \leqslant-\left(b G_{4,4}^{\frac{2+\omega}{m_{5}}}+G_{3,3}^{\frac{2+\omega}{m_{4}}}+\right. \\
\left.a_{22} G_{2,2}^{\frac{2+\omega}{m_{3}}}+G_{1,1}^{\frac{2+\omega}{m_{2}}}\right) .
\end{gathered}
$$

Based on Assumption 1, (43) could be translated into

$$
\dot{Q}_{1}(e) \leqslant-\sum_{j=1}^{n} \phi_{j}\left|G_{j, j}\right|^{\frac{2+\omega}{m_{j+1}}} \leqslant-\sigma \varphi W(e)
$$

where $\phi_{1}=1, \phi_{2}=a_{22}, \phi_{3}=1, \phi_{4}=b, W(e)=$ $\sum_{j=1}^{n}\left|e_{j+1}-e_{j}^{\frac{m_{j+1}}{m_{j}}}\right|^{\frac{2+\omega}{m_{j+1}}}$.

Based on the definition of homogeneity, one can conclude availably that the Lyapunov candidate function $Q_{1}(e)$ is homogeneous of degree 2 with dilations $\left(m_{1}, \ldots, m_{4}\right)$. Similarly, $W(e)$ is also homogeneous of degree $\omega+2$ for identical dilations. The following inequality holds, when the appropriate parameter $G_{1}>0$ can be selected.

$$
\dot{Q}_{1} \leqslant-\sigma W \leqslant-\sigma G_{1} Q_{1}^{\frac{2+\omega}{2}}=-G_{2} Q_{1}^{\frac{2+\omega}{2}}
$$

where $G_{2}=\sigma G_{1} \cdot Q_{1}(e)$ will converge to zeros in a finite time. In other words, the errors of system converge rapidly to the equilibrium point of system (12).

(ii) Under the previous conclusion, the strict proof process of the high-order FTSO for the system (5) would be verified.

Above all, the following error dynamics of the highorder FTSO could get

$$
\left\{\begin{aligned}
\dot{\bar{e}}_{1}= & L \bar{e}_{2}-L k_{1} \bar{e}_{1}^{m_{2}} \\
\dot{\bar{e}}_{2}= & a_{22} L \bar{e}_{3}-a_{22} L k_{2} \bar{e}_{1}^{m_{3}}+ \\
& \frac{f_{2}\left(t, x_{1}, x_{2}, u\right)-f_{2}\left(t, x_{1}, \hat{x}_{2}, u\right)}{L} \\
\dot{\bar{e}}_{3}= & L \bar{e}_{4}-L k_{3} \bar{e}_{1}^{m_{4}}+ \\
& \frac{f_{3}\left(t, x_{1}, x_{2}, x_{3}, u\right)-f_{3}\left(t, x_{1}, \hat{x}_{2}, \hat{x}_{3}, u\right)}{L^{2}} \\
\dot{\bar{e}}_{4}= & -b L k_{4} \bar{e}_{1}^{m_{5}}+\frac{f_{4}\left(t, x_{1}, x_{2}, x_{3}, x_{4}, u\right)}{L^{3}}- \\
& \frac{f_{4}\left(t, x_{1}, \hat{x}_{2}, \hat{x}_{3}, \hat{x}_{4}, u\right)}{L^{3}}
\end{aligned}\right.
$$

where $\bar{e}_{i}=\left(x_{i}-\hat{x}_{i}\right) / L^{i-1}, i=1, \ldots, 4$. 
Under the nonlinear mapping (9), system (46) can be rewritten as

$$
\dot{\boldsymbol{e}}=L\left[\begin{array}{c}
c_{1}\left(e_{2}-e_{1}^{m_{2}}\right) \\
a_{22} c_{2}\left(e_{3}-e_{1}^{m_{3}}\right) \\
c_{3}\left(e_{4}-e_{1}^{m_{4}}\right) \\
b c_{4}\left(-e_{1}^{m_{5}}\right)
\end{array}\right]+\left[\begin{array}{c}
0 \\
\frac{f_{2}-\hat{f}_{2}}{k_{1} L} \\
\frac{f_{3}-\hat{f}_{3}}{k_{2} L^{2}} \\
\frac{f_{4}-\hat{f}_{4}}{k_{3} L^{3}}
\end{array}\right]
$$

where $\hat{f}_{i}:=f_{i}\left(t, x_{1}, \hat{x}_{2}, \ldots, \hat{x}_{i}, u\right)$ for $i=2,3,4$. Based on the previous fundamental work of $Q_{1}(e)$ and (45), it is clear that

$$
\begin{gathered}
\left.\dot{Q}_{1}\right|_{(47)} \leqslant-L \sigma \varphi W+ \\
\sum_{i=2}^{4} \frac{\partial Q_{1}(e)}{\partial e_{i}} \frac{f_{i}-\hat{f}_{i}}{k_{i-1} L^{i-1}} .
\end{gathered}
$$

Under Assumptions 1 and 2, one can acquire that

$$
\begin{gathered}
\frac{\left|f_{i}-\hat{f}_{i}\right|}{k_{i-1} L^{i-1}} \leqslant \frac{a_{i} \phi_{i}(t, z) \sum_{j=2}^{i}\left|k_{j-1} L^{j-1} e_{j}\right|^{\left(m_{i}+\tau\right) / m_{j}}}{k_{i-1} L^{i-1}} \leqslant \\
\varepsilon \phi(z) \sum_{k=2}^{i}\left|e_{k}\right|^{\frac{m_{i}+\tau}{m_{k}}}
\end{gathered}
$$

where $L \geqslant 1$ and $\varepsilon>0$. Integrating (48) with (49) yields

$$
\begin{gathered}
\left.\dot{Q}_{1}\right|_{(47)} \leqslant-L \sigma \phi(z) W+ \\
\varepsilon \phi(z) \sum_{i=2}^{4}\left[\left|\frac{\partial Q_{1}(e)}{\partial e_{i}}\right| \sum_{k=2}^{i}\left|e_{k}\right|^{\frac{m_{i}+\omega}{m_{k}}}\right] .
\end{gathered}
$$

Based on the homogeneous definition, one can conclude that this term $\left|\frac{\partial Q_{1}(e)}{\partial e_{i}}\right| \sum_{k=2}^{i}\left|e_{k}\right|^{\frac{m_{i}+\omega}{m_{k}}}$ is homogeneous with a degree of $\omega+2$. The following inequality holds, when the appropriate parameter $G_{3}$ can be selected.

$$
\begin{gathered}
\left.\dot{Q}_{1}\right|_{(47)} \leqslant-L \underline{\sigma} \phi(y) W(e)+ \\
\varepsilon \phi(y) G_{3} W(e) .
\end{gathered}
$$

The derivative of $Q_{1}$ would be negatively definite, when the condition $L \sigma>\varepsilon G_{3}$ is met. In addition, the equation (51) could be translated into

$$
\left.\dot{Q}_{1}\right|_{(18)} \leqslant-\left(L \underline{\sigma}-\varepsilon G_{3}\right) a / \underline{\sigma} G_{1} Q_{1}^{\frac{2+\omega}{2}} .
$$

Therefore, the error dynamic system (47) or (46) is globally finite-time stable.

\subsection{Composited IGC algorithm}

Theorem 2 On the basis of the high-order FTSO (6), the proposed composited IGC algorithm is

$$
\left\{\begin{array}{l}
u=\frac{1}{b}\left(-v_{4} s_{4}-v_{41} \operatorname{sig}^{r}\left(s_{4}\right)-f_{4}+\dot{x}_{4 c}\right) \\
s_{4}=\hat{x}_{4}-x_{4 c} \\
\tau_{4} \dot{x}_{4 c}+x_{4 c}=x_{4 c}^{*}, x_{4 c}(0)=x_{4 c}^{*}(0) \\
x_{4 c}^{*}=-v_{3} s_{3}-v_{31} \operatorname{sig}^{r}\left(s_{3}\right)-f_{3}+\dot{x}_{3 c} \\
s_{3}=\hat{x}_{3}-x_{3 c} \\
\tau_{3} \dot{x}_{3 c}+x_{3 c}=x_{3 c}^{*}, x_{3 c}(0)=x_{3 c}^{*}(0) \\
x_{3 c}^{*}=\frac{1}{a_{22}}\left(-v_{2} s_{2}-v_{21} \operatorname{sig}^{r}\left(s_{2}\right)-f_{2}+\dot{x}_{2 c}\right) \\
s_{2}=\hat{x}_{2}-x_{2 c} \\
\tau_{2} \dot{x}_{2 c}+x_{2 c}=x_{2 c}^{*}, x_{2 c}(0)=x_{2 c}^{*}(0) \\
x_{2 c}^{*}=-v_{1} s_{1}-v_{11} \operatorname{sig}^{r}\left(s_{1}\right) \\
s_{1}=\hat{x}_{1}-\Delta
\end{array}\right.
$$

which could obtain the desired endgame performance, in spite of the inaccuracy measurement of the LOS rate, where $v_{i}>0, v_{i 1}>0$ and $0<r<1$ for $i=1, \ldots, n$.

\section{Proof}

Above all, the following Lyapunov candidate function is adopted:

$$
V_{s}=\sum_{i=1}^{4} \frac{1}{2} s_{i}^{2} .
$$

Taking the derivative of $V_{s}$ and integrating with system (53), one can acquire that

$$
\begin{gathered}
\dot{V}_{s}=\sum_{i=1}^{4} s_{i} \dot{s}_{i}=\sum_{i=1}^{4} s_{i}\left(-v_{i} s_{i}-v_{i 1} \operatorname{sig}^{\varepsilon}\left(s_{i}\right)\right)= \\
-\left(\sum_{i=1}^{4} v_{i} s_{i}^{2}\right)-\left(\sum_{i=1}^{4} v_{i 1}\left|s_{i}\right|^{\varepsilon+1}\right) \leqslant-\sum_{i=1}^{4} v_{i 1}\left|s_{i}\right|^{\varepsilon+1} .
\end{gathered}
$$

Under Lemma 4, it is obvious that

$$
\begin{gathered}
-\sum_{i=1}^{4} v_{i 1}\left|s_{i}\right|^{\varepsilon+1}=-\sum_{i=1}^{4} v_{i 1}\left|s_{i}\right|^{2 \cdot \frac{\varepsilon+1}{2}} \leqslant \\
-v_{\min } \sum_{i=1}^{4}\left(s_{i}^{2}\right)^{\frac{\varepsilon+1}{2}} \leqslant-v_{\min }\left(\sum_{i=1}^{4} s_{i}^{2}\right)^{\frac{\varepsilon+1}{2}}= \\
-v_{\min }\left(2 V_{s}\right)^{\frac{\varepsilon+1}{2}}=-2^{\frac{\varepsilon+1}{2}} v_{\min } V_{s}^{\frac{\varepsilon+1}{2}}
\end{gathered}
$$

where $0<\varepsilon<1, v_{\min }=\min \left\{v_{11}, v_{21}, v_{31}, v_{41}\right\}$.

Substituting (56) into (55) yields

$$
\dot{V}_{s}+2^{\frac{\varepsilon+1}{2}} v_{\min } V_{s}^{\frac{\varepsilon+1}{2}}<0
$$

which could guarantee the system (53) variables globally finite-time stable. 


\section{Numerical simulation}

Performance of the composited IGC algorithm is surveyed by means of numerical simulation in this section. First, we provide a comparison between the composited IGC and the traditional IGC, when the LOS rate is mixed with disturbance. Next, the robustness of the proposed algorithm against parameter disturbance is investigated. Finally, its flexibility in existence of small-range target maneuver is evaluated.

The initial states and constraint condition of simulation are shown in Table 1 and Table 2, respectively.

\begin{tabular}{cc} 
Table $\mathbf{1}$ Initial state of simulation \\
\hline State variable & Value \\
\hline Simulation $\mathrm{step} / \mathrm{s}$ & 0.001 \\
Position of vehicle/m & {$[0,16000]$} \\
Velocity of vehicle $/(\mathrm{m} / \mathrm{s})$ & 1800 \\
Position of target/m & {$[0,32000]$} \\
Velocity of target $/(\mathrm{m} / \mathrm{s})$ & 0 \\
\hline & \\
Table 2 & Constraint condition of simulation \\
\hline Constraint index & Range \\
\hline Terminal precision $/ \mathrm{m}$ & $(0,10]$ \\
Amplitude of Angle of attack $/\left(^{\circ}\right)$ & {$[0,12.5]$} \\
Amplitude of deflection $/\left({ }^{\circ}\right)$ & {$[-30,30]$} \\
Rate of deflection $/\left({ }^{\circ} / \mathrm{s}\right)$ & {$[-50,50]$}
\end{tabular}

Case 1 Performance comparison with inaccuracy information of LOS rate

One of the innovative points in this paper is to design the integrated algorithm with the inaccuracy information of the LOS rate. Thus, the traditional IGC algorithm (TIGC) based on back-stepping and dynamic inverse is employed to validate the advantages of the proposed FTSObased IGC (FTSO-IGC), while the LOS rate is mixed with the disturbance. Assume that the mixed disturbance is $5 \times 10^{-3} \sin (0.2 t) \mathrm{rad}$. The contrastive simulation curves of flight trajectory, virtual control and system input are plotted in Figs. 1-4, respectively.

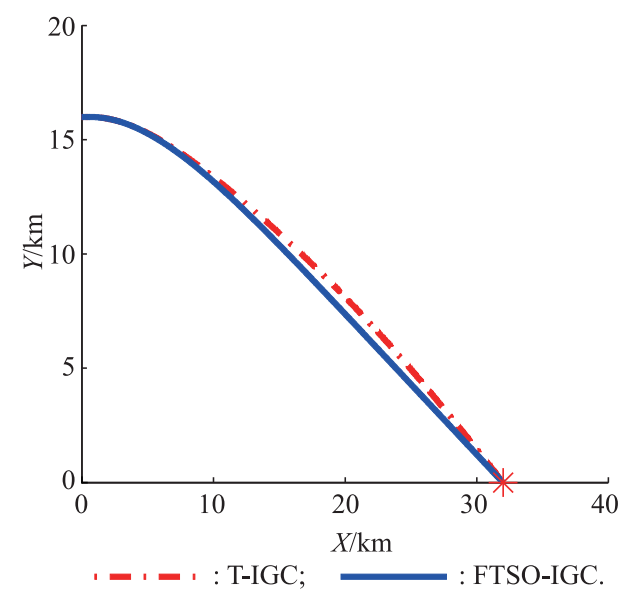

Fig. 1 Trajectory of the vehicle

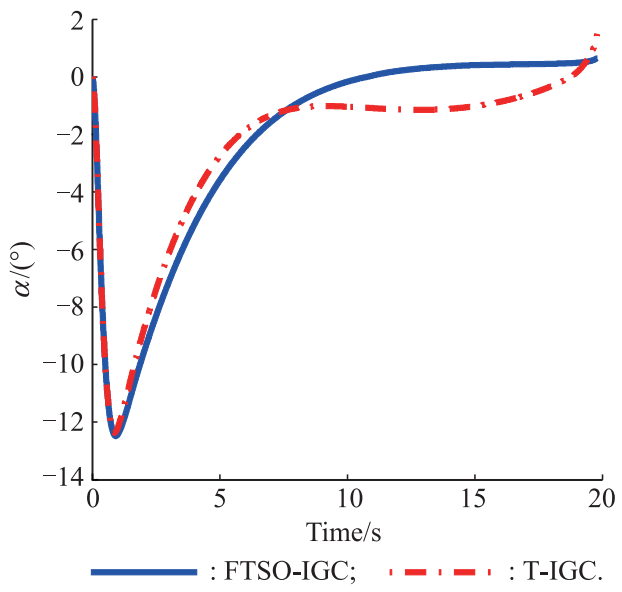

Fig. 2 Curves of $\alpha$

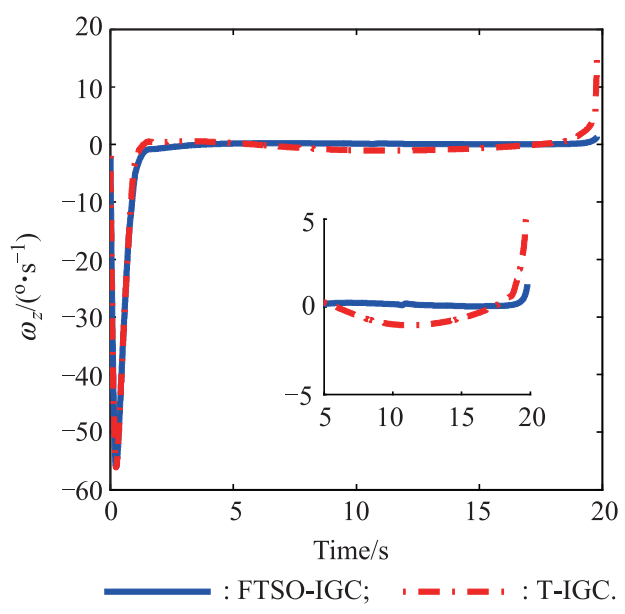

Fig. 3 Curves of $\omega_{z}$

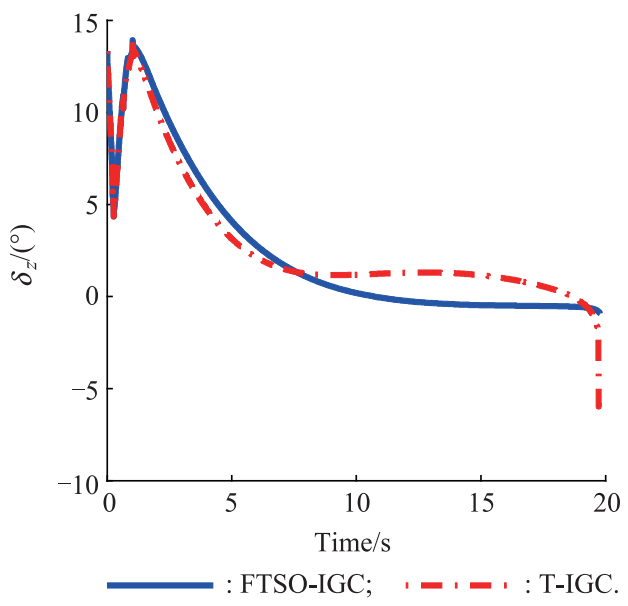

Fig. 4 Curves of $\delta_{z}$

From Figs. 1-4, it is obvious that both the T-IGC and the proposed FTSO-IGC can ensure an ideal strike precision and the terminal precisions are $2.792 \mathrm{~m}$ and $0.021 \mathrm{~m}$, respectively. In addition, the virtual control and system input are sufficiently glazed and various involved physical constraints are met. However, the effect of disturbance on 
the system is gradually increasing with the decrease of the LOS rate.

Compared with FTSO-IGC, the additional $\alpha, \omega_{z}$ and $\delta_{z}$ of the T-IGC algorithm are required to impel the LOS rate to converge to zero. Consequently, the proposed FTSOIGC attenuates the design conservation of the flight control system and reduces excessive control.

The actual state variables, their corresponding estimations and the errors of the observer are depicted in Fig. 5. As shown in Fig.5, one can conclude that the errors of the FTSO are capable of rapid convergence to a sufficiently small region around zero. That is to say, the developed high-order FTSO is provided with satisfactory performance of estimating the state variables, which can improve the damage performance ability of the vehicle.

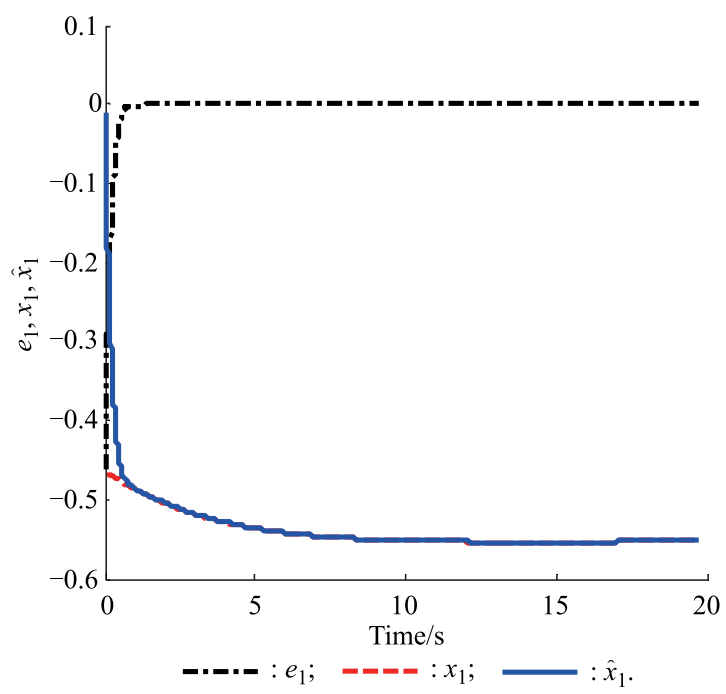

(a) $\lambda_{D}$

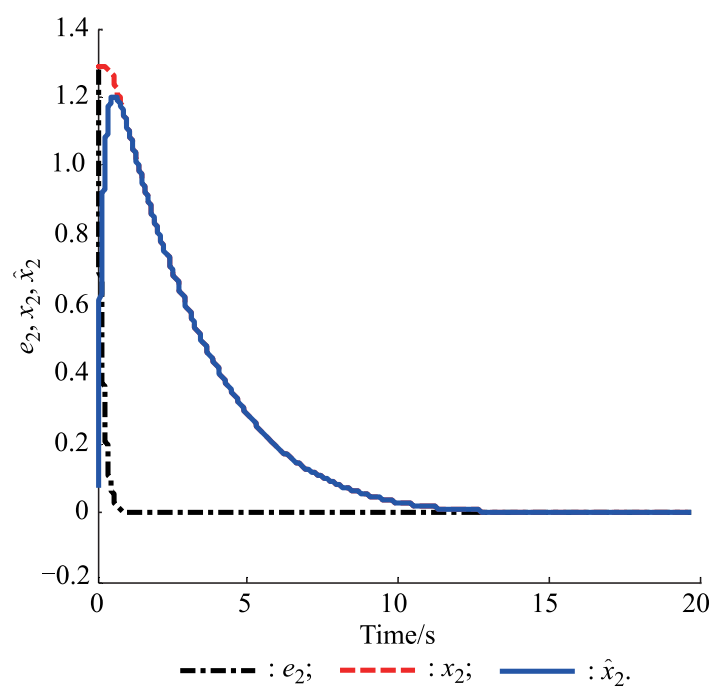

(b) $\dot{\lambda}_{D}$

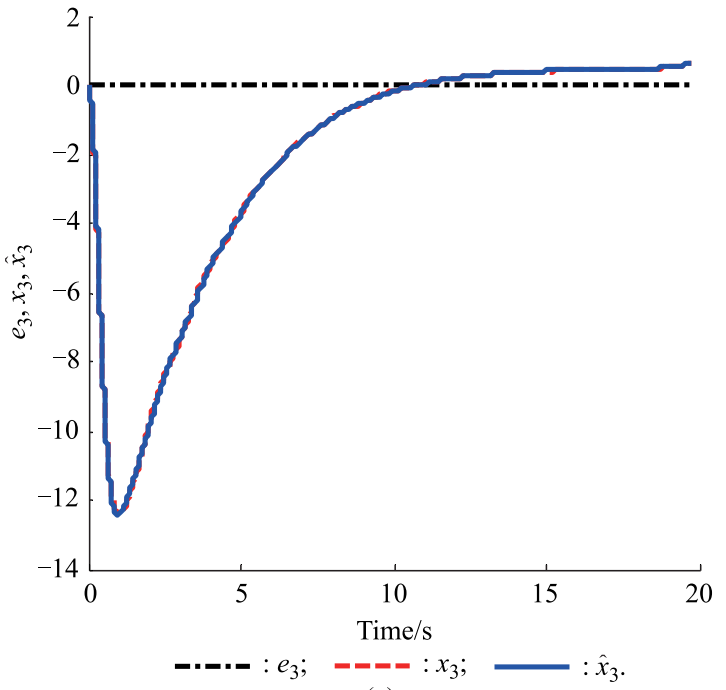

(c) $\alpha$

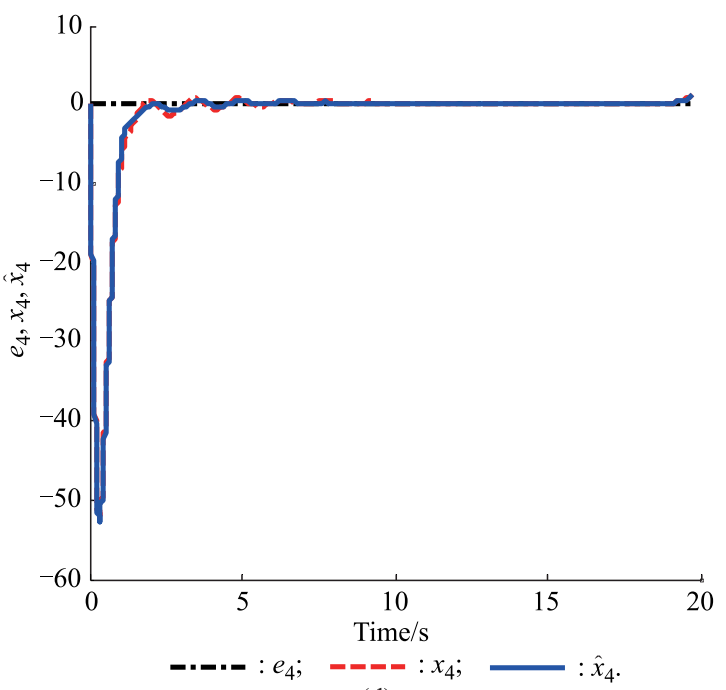

(d) $\omega_{z}$

Fig. 5 Curves of the actual states, estimations and errors of the observer

Case 2 Robustness evaluation against aerodynamic coefficient uncertainty

Modern vehicles usually need to accomplish the specific flight task in complex atmospheric environment. Wherefore, the propsed composited IGC must possess a weak sensitivity to the aerodynamic coefficient. Let aerodynamic coefficient uncertainty subject to the following distribution:

$$
\Omega \sim T(-0.2,0.2)
$$

where $T$ represents the uniform distribution.

The generated experimental design scheme based on the Monte Carlo method serves as the additional bias term, which is superposed on the nonimal aerodynamic coefficient in the emulation experiments. The multigroup curves are displayed in Figs. 6-9. Despite the bias term of the 
aerodynamic coefficient varying randomly within $[-20 \%$, $20 \%$ ], the design index of the terminal precision is satisfied. Moreover, the virtual control variables $\alpha, \omega_{z}$ and the actual system input $\delta_{z}$ all tend to be stable in the end.

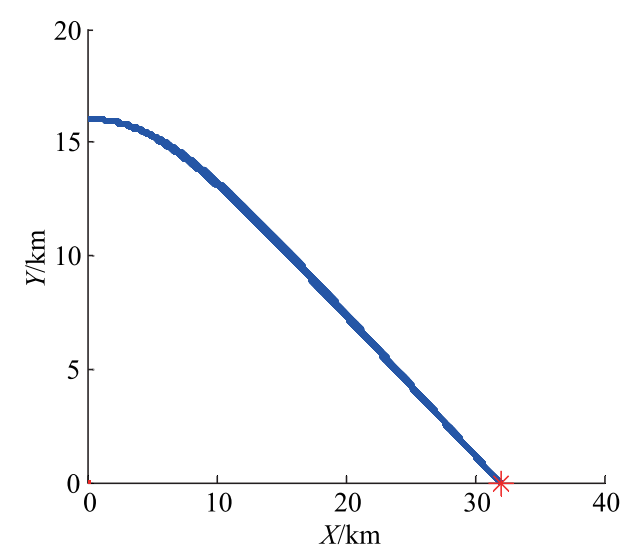

Fig. 6 Trajectory of vehicle with aerodynamic coefficient uncertainty

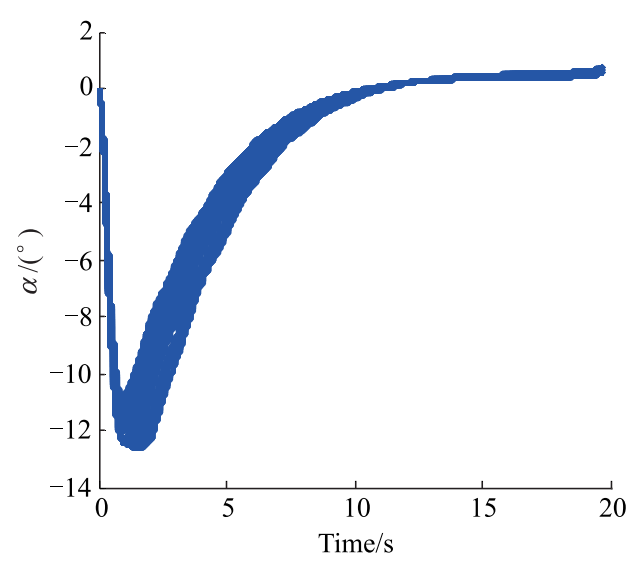

Fig. 7 Curves of $\alpha$ with aerodynamic coefficient uncertainty

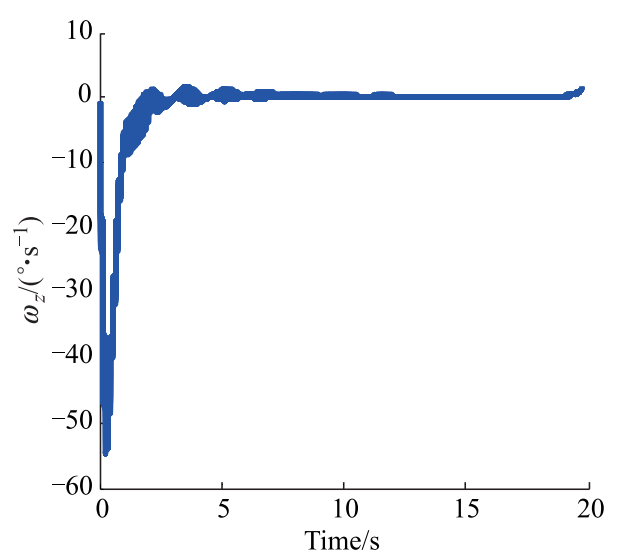

Fig. 8 Curves of $\omega_{z}$ with aerodynamic coefficient uncertainty

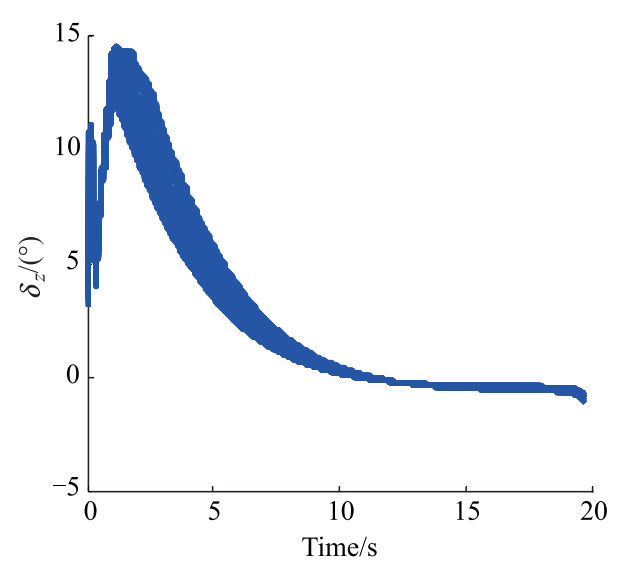

Fig. 9 Curves of $\delta_{z}$ with aerodynamic coefficient uncertainty

A noteworthy fact is that the random bias term of aerodynamic environment affects greatly on the amplitude of $\alpha$ and $\omega_{z}$. The aerodynamic coefficient becomes larger, when the bias term is greater than zero. As a result, the required amplitude of $\alpha$ and $\delta_{z}$ will diminish to generate the specific aerodynamic lift. Thus, the composited IGC method possesses a weak sensitivity to the aerodynamic coefficient uncertainty.

Case 3 Robustness evaluation against small-range target maneuver

In this part, our goal is to test the robustness of the developed algorithm in the presence of small-range target maneuver. We set the position of the target in the nominal case as the original point, and then perform the simulation experiments of target maneuver at different speeds along different directions. The climbing maneuver is defined as the situation where the inclination of the target's speed is $45^{\circ}$ or $135^{\circ}$. The target maneuver is defined as the error of the LOS rate, caused by measurement or computation. The relevant experimental conditions are shown in Table 3.

Table 3 Maneuvering condition of target

\begin{tabular}{ccc}
\hline Index & Inclination $/\left({ }^{\circ}\right)$ & Velocity $/(\mathrm{m} / \mathrm{s})$ \\
\hline 1 & 0 & 25 \\
2 & 0 & 50 \\
3 & 45 & 25 \\
4 & 45 & 50 \\
5 & 135 & 25 \\
6 & 135 & 50 \\
7 & 180 & 25 \\
8 & 180 & 50 \\
\hline
\end{tabular}

According to the experimental results, we find that the distance between the vehicle and the target is always within the given precision. The trajectories of the vehicle in different situations are shown in Fig. 10. As we can see from the experimental results, when the targets are doing lowspeed maneuver in different directions, the vehicle can hit them precisely with the proposed FTSO-based IGC algorithm. This has demonstrated the efficiency of the designed 
FTSO, which is able to obtain the information of the LOS rate accurately and quickly.

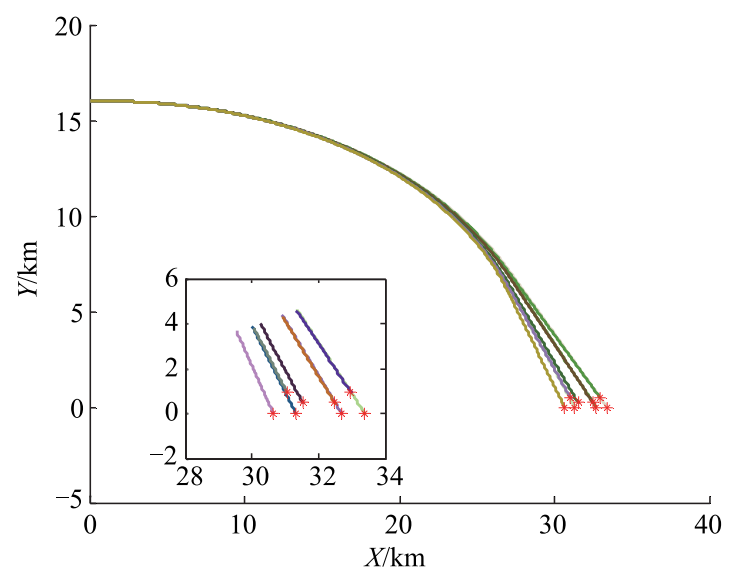

Fig. 10 Trajectory curves of vehicle with maneuvering targets

\section{Conclusions}

In this paper, the composited FTSO-based IGC algorithm is proposed for the BTT vehicle in the dive phase, via the fusion of back-stepping and dynamic inverse. The highorder FTSO can make full use of the information of system to estimate the states of the system. The errors of the observer can converge rapidly to a sufficiently small region around zero and the difficulty caused by the inaccuracy measurement of the LOS rate is successfully overcome. Compared with the traditional IGC algorithm, the composited IGC method can attenuate effectively the design conservation and reduce excessive control. Besides, it is also robust to aerodynamic coefficient uncertainty and small-range target maneuver.

\section{References}

[1] ZHANG Y, GUO J, TANG S J, et al. Integrated missile guidance and control three-channel decoupling design method. Acta Aeronautica et Astronautica Sinica, 2014, 35(12): 3438 3450. (in Chinese)

[2] ZHA X, CUI P Y, CHANG B J. An integrated approach to guidance and control for aircraft applying to attack the ground fixed targets. Journal of Astronautics, 2005, 26(1): 13 - 18. (in Chinese)

[3] YIN Y X, YANG M, WANG Z C. Three dimensional guidance and control for missile. Electric Machines and Control, 2010, 14(3): 83 -91. (in Chinese)

[4] ZHOU H, ZHAO H, HUANG H Q, et al. Integrated guidance and control design of the suicide UCAV for terminal attack. Journal of Systems Engineering and Electronics, 2017, 28(3): $546-555$

[5] HE S M, SONG T, LIN D F. Impact angle constrained integrated guidance and control for maneuvering target interception. Journal of Guidance, Control, and Dynamics, 2017, 40(10): $2652-2660$.

[6] HE S M, WANG W, WANG J. Three-dimensional multivariable integrated guidance and control design for maneuvering targets interception. Journal of The Franklin Institute, 2016, 353(16): 4330-4350.

[7] TIAN B L, FAN W R, ZONG Q. Integrated guidance and control for reusable launch vehicle in reentry phase. Nonlinear Dynamic, 2015, 80(1/2): $397-412$.

[8] SUN X Y, CHAO T, WANG S Y, et al. Integrated guidance and control design method considering channel coupling. Journal of Astronautics, 2016, 37(8): 936-945. (in Chinese)

[9] XIN M, BALAKRISHNAN S N, OHLMEYER E J. Integrated guidance and control of missile with $\theta$-D method. IEEE Trans. on Control Systems Technology, 2006, 14(6): 981-992.

[10] MENON P K, OHLMEYER E J. Integrated design of agile missile guidance and autopilot system. Control Engineering Practice, 2001, 9(10): 1095-1106.

[11] PANCHAL B, MATE N, TALOLE S E. Continuous-time predictive control-based integrated guidance and control. Journal of Guidance, Control, and Dynamics, 2017, 40(7): 15791595.

[12] YAN H, TAN S P, HE Y Z. A small-gain method for integrated guidance and control in terminal phase of reentry. Acta Astronautica, 2017, 132: 282-292.

[13] VADDI S, MENON P K, OHLMEYER E J. Numerical statedependent Riccati equation approach for missile integrated guidance control. Journal of Guidance, Control, and Dynamics, 2009, 32(2): 699-703.

[14] DONG F Y, LEI H M, ZHOU C J, et al. Research of integrated robust high order sliding mode guidance and control for missile. Acta Aeronautica et Astronautica Sinica, 2013, 34(9): 2212-2218. (in Chinese)

[15] DONG F Y, LEI H M, LI J, et al. Design of integrated adaptive optimal sliding-mode guidance and control for interceptor. Journal of Astronautics, 2013, 34(11): 1456-1641. (in Chinese)

[16] SONG H T, ZHANG T. Fast robust integrated guidance and control design of interceptors. IEEE Trans. on Control Systems Technology, 2016, 24(1): 349-356.

[17] ZHANG C, WU Y J. Non-singular terminal dynamic surface control based integrated guidance and control design and simulation. ISA Transactions, 2016, 63: $112-120$.

[18] WANG J H, LIU L H, ZHAO T, et al. Integrated guidance and control for hypersonic vehicle in dive phase with multiple constraints. Aerospace Science and Technology, 2016, 53: $103-$ 115.

[19] WANG X, WANG J. Partial integrated missile guidance and control with finite time convergence. Journal of Guidance, Control, and Dynamics, 2013, 36(5): 1399 - 1409.

[20] SUN X J, ZHOU R, HOU D L. Output-feedback based partial integrated missile guidance and control law design. Journal of Systems Engineering and Electronics, 2016, 27(6): $1238-$ 1248.

[21] SUN X Y, WANG S Y, SHENG S J, et al. Adding a power integrator technique based integrated guidance and control design. Control and Decision, 2018, 33(2): 242-248. (in Chinese)

[22] SHIMA T, IDAN M, ODED M G. Sliding-mode control for integrated missile autopilot guidance. Journal of Guidance, Control, and Dynamics, 2006, 29(2): 250-260.

[23] YAN H, JI H B. Integrated guidance and control for dualcontrol missile based on small gain theorem. Automatica, 2012, 48(10): 2686-2692.

[24] HOU M Z, DUAN G R. Adaptive dynamic surface control for integrated missile guidance and autopilot. International Journal of Automation and Computing, 2011, 8(1): 122-127.

[25] GURFIL P. Zero-miss-distance guidance law based on lineof-sight rate measurement only. Control Engineering Practice, 
2003, 11(7): $819-832$.

[26] CHWA D, CHOI J Y. Adaptive nonlinear guidance law considering control loop dynamcis. IEEE Trans. on Aerospace and Electronic Systems, 2003, 39(4): $1134-1143$.

[27] BHAT S P, BERNSTEIN D S. Finite-time stability of continuous autonomous systems. SIAM Journal on Control and Optimization, 2000, 38(3): $751-766$.

[28] DING S H, LI S H, ZHENG W X. Nonsmooth stabilization of a class of nonlinear cascaded systems. Automatica, 2012, 48(10): $2597-2606$.

[29] DU H B, QIAN C J, YANG S Z, et al. Recursive design of finite-time convergent observers for a class of time-varying nonlinear system. Automatica, 2013, 49(2): 601-609.

[30] HUANG S, XIANG Z. Finite-time stabilization of switched stochastic nonlinear systems with mixed odd and even powers. Automatica, 2016, 73: 130-137.

[31] SUN S. Guidance laws with finite time convergence for homing missiles. Harbin, China: Harbin Institute of Technology, 2010. (in Chinese)

[32] BHAT S P, BERNSTEIN D S. Continuous finite-time stabilization of the translational and rotational double integrators. IEEE Trans. on Automatic Control, 1998, 43(5): 678-682.

[33] QIAN C, LIN W. A continuous feedback approach to global strong stabilization of nonlinear systems. IEEE Trans. on Automatic Control, 2001, 46(7): $1061-1079$.

[34] BACCIOTTI A, ROSIER L. Liapunov functions and stability in control theory. Automatica, 2005, 41(12): 2183-2184.

\section{Biographies}

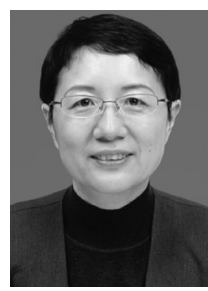

MA Ping was born in 1970. She received her Ph.D. degree in control science and engineering from Harbin Institute of Technology in 2003. She is currently a professor in the Control and Simulation Center at Harbin Institute of Technology. Her research interests are the design and implement ation of distributed simulation system and verification validation and accreditation of complex simulation system.

E-mail: pingma@hit.edu.cn

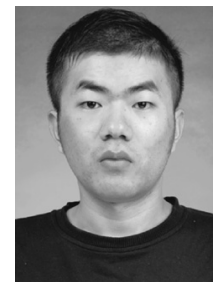

ZHANG Denghui was born in 1992. He received his M.S. degree in automation from Harbin Institute of Technology in 2016. He is now a Ph.D. degree candidate in the Control and Simulation Center at Harbin Institute of Technology. His research interests are navigation, guidance and control.

E-mail: zdh_hit_2016@163.com

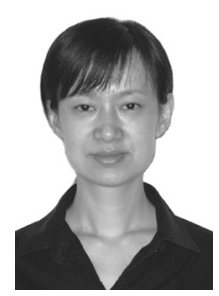

WANG Songyan was born in 1976. She received her Ph.D. degree in navigation, guidance and control from Harbin Institute of Technology in 2007. She is currently an associate professor in the Control and Simulation Center at Harbin Institute of Technology. Her research interests include navigation, guidance and control and performance evaluation.

E-mail: sywang@hit.edu.cn

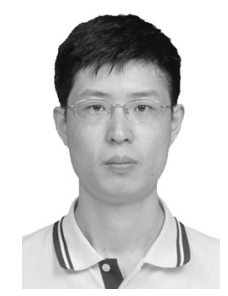

CHAO Tao was born in 1983. He received his Ph.D. degree in navigation, guidance and control from Harbin Institute of Technology in 2011. He is currently an associate professor in the Control and Simulation Center at Harbin Institute of Technology. His research interests include navigation, guidance and control and performance evaluation.

E-mail: chaotao2000@163.com 